Article

\title{
A Study on the Heat Transfer of Surrounding Rock-Supporting Structures in High-Geothermal Tunnels
}

\author{
Mingnian Wang ${ }^{1,2}$, Yunpeng $\mathrm{Hu}^{1,2}$, Dagang Liu ${ }^{1,2, *}$, Cheng Jiang ${ }^{1,2}$, Qiling Wang ${ }^{1,2, *}$ and \\ Yicheng Wang ${ }^{1,2}$ \\ 1 School of Civil Engineering, Southwest Jiaotong University, Chengdu 610031, Sichuan, China \\ 2 Key Laboratory of Transportation Tunnel Engineering, Ministry of Education, Southwest Jiaotong University, \\ Chengdu 610031, Sichuan, China \\ * Correspondence: ldg@home.swjtu.edu.cn (D.L.); wql199486@my.swjtu.edu.cn (Q.W.)
}

Received: 1 March 2020; Accepted: 24 March 2020; Published: 27 March 2020

\begin{abstract}
The temperature distribution is one of the most vital parameters which should be fully considered in high geothermal tunnel design. For the purpose of studying the impact of temperature disturbance caused by construction on temperature distribution of surrounding rock and lining structure in a high geothermal tunnel, a new finite difference model for temperature prediction was proposed. Based on the abundant field test results, forecast analysis for the research of a high geothermal tunnel in this paper is made. The results indicate that the temperature of the surrounding rock near the tunnel sidewall decreases obviously in the first 14 days while that of the surrounding rock far away is stable after tunnel excavation, and the rock temperature showed three ways of change: undulate type $(<2 \mathrm{~m})$, decline type $(2-5 \mathrm{~m})$ and stable type $(>5 \mathrm{~m})$. There is a linear relationship between the initial rock temperature and the released heat of the surrounding rock. The radius of the heat-adjusting layer and the initial rock temperature presents a quadratic function relation. The lining concrete actually cures under the variable high-temperature environment and the real curing temperature decreases with time and becomes stable seven days later. The results would contribute to providing support for high geothermal tunnel research and design.
\end{abstract}

Keywords: high geothermal tunnel; temperature test; finite difference model; variable temperature

\section{Introduction}

With the rapid development of traffic engineering under complex geological conditions, there is a rapid growth in the number of tunnels constructed worldwide under high geothermal activity conditions recently, such as Gotthard-Basis Tunnel in Switzerland Swit, Apennine Railway Tunnel in Italy, Sangzhuling Railway Tunnel in China and so on [1,2]. High geothermal is an unavoidable and widely distributed problem in deep, long tunnel constructions. It may cause an increase of thermal stress and the degradation of the material properties of concrete [3], resulting in the failure of the lining structure in the tunnel [4-6]. Moreover, a high geothermal environment [7] also puts tunnel workers under terrible working conditions [8-10]. Therefore, the temperature fields of the surrounding rock and lining concrete become an urgent important issue to study to solve the high temperature-resistant problems in high geothermal tunnels.

Previous research on the temperature field in tunnel engineering mostly focused on the cold-region tunnel. Zhang et al. [11] concluded that the flow of groundwater had a significant impact on the distribution of temperature of the surrounding rock by an experimental model test. He et al. [12] tested the air temperature, wind force conditions and atmospheric pressure on the spot and proposed a new 
prediction model of freezing-thawing conditions concerning the combined convection-conduction in the rock surrounding tunnels in cold regions. Zhang et al. [13] did research on the three-dimensional temperature properties in cold-region tunnels by the finite element method. Lai et al. [14] proved that the freezing-thawing behaviors of the rock surrounding a tunnel can be correctly predicted even if the air temperature along the tunnel is unknown. According to the heat transfer theory, Lai et al. researched temperature, seepage and stress fields in cold-region tunnels and they also made nonlinear analyses of temperature distribution using finite element formulas. Zeng et al. [15] studied that it has an adverse effect on the freezing damage in the tunnel when the mechanical ventilation velocity was higher than natural velocity. The negative ventilation direction is helpful to alleviate freezing damage. Zhou et al. [16] predicted the temperature field of a railway tunnel. The study results demonstrated that the mechanical ventilation winds and train-induced winds should be considered in the engineering design of tunnels in cold regions, which has a significant impact on the distribution of the temperature field in the surrounding rock.

In the aspect of high-temperature tunnels, the study results are mainly based on the influence of fire and smoke on the tunnel temperature field. The previous studies indicated that the initial fuel temperature significantly influences the flame spread behaviors over the liquid fuels and the temperature field in tunnels. Chuangang et al. [17] investigated the typical smoke characteristic parameters, such as temperature distribution, and researched the impact of different fire positions on natural ventilation effectiveness in a tunnel by large eddy simulation [18]. The smoke layer interface height was also determined by conducting a 1/6th scale tunnel model test in medium-scale tunnel fire scenarios [19]. Tian et al. [20] showed that the smoke concentration significantly influenced the ceiling temperature. Based on the theoretical analysis and the curve fits of the tunnel ceiling temperature decay with distance from the fire source, a model for the ceiling temperature decline along the tunnel was proposed. Liang et al. [21] investigated the distribution of temperature in tunnel fires by experimental tests and theoretical analyses. The factors that influence the smoke temperature distribution are discussed according to the equation they established.

In summary, previous researchers have a provided number of valuable results on the temperature field in tunnel constructions. However, very limited research findings are available on the high geothermal tunnel $[22,23]$. Most studies of temperature field under hot environments lack on-site data for support and the existing theory about temperature distribution of the surrounding rock has not been verified, especially the characteristics of high temperature on field. Therefore, this paper aims to investigate the temperature distribution and heat transfer characteristics of the surrounding rock, primary support and secondary lining during the construction progress by conducting an on-site test under a hot environment in a tunnel and theoretical analysis. A model for predicting the temperature of the surrounding rock-lining structure was obtained based on both the MATLAB simulation and the finite difference calculations, and was verified by the field test results. The research results may provide useful information and theoretical support for ventilation and cooling, the design of support structure and the safety of workers in a high geothermal tunnel.

\section{Establishment of Finite Difference Model}

\subsection{Fundamental Assumptions and Governing Equations}

The purpose of this study was to investigate the temperature distinguishing feature in a high geothermal tunnel during the construction procession. Therefore, for simplicity, the one-dimensional unsteady heat transfer analysis of the cross-section of the high-temperature tunnel in this paper is based on the following assumptions:

(1) The tunnel cross-section is simplified to a circular shape [2,24].

(2) The tunnel lining and the surrounding rock are isotropic, and the thermal properties are stable and unaffected by temperature. 
(3) The heat transfer conditions in the circumferential direction of the inner wall of the tunnel are the same, and the air temperature in the tunnel is uniform and constant.

(4) There is no contact thermal resistance between the layers of the tunnel.

(5) The heat transferring along the axis is much smaller than that along the radial direction, assuming that heat is only transferred in the radial direction, and there is no heat flow in the axial and circumferential directions.

(6) There is no internal heat source in the medium of each layer of the tunnel. The heat transferring mode between the tunnel lining and surrounding rock is heat conduction and that between the lining and the air in the tunnel is heat convection. Heat radiation is neglected because of its relatively small magnitude [12].

One-dimensional unsteady heat conduction [25] differential equations of the temperature field between lining-surrounding and rocks in polar coordinates can be written as [14]:

$$
\frac{\partial t_{k}}{\partial \tau}=a_{k}\left(\frac{1}{r} \frac{\partial t_{k}}{\partial r}+\frac{\partial^{2} t_{k}}{\partial r^{2}}\right)
$$

where $a_{k}$ and $t_{k}$, respectively, represent the temperature coefficient and temperature of the k-th layer medium. $\tau$ is the time. $r$ is the distance from the tunnel axis.

$$
a_{k}=\frac{\lambda_{k}}{c_{k} \rho_{k}}
$$

where $\lambda_{k}, c_{k}$ and $\rho_{k}$, respectively, represent thermal conductivity, specific heat capacity and intensity.

The initial conditions are,

$$
t(\tau, r)=t_{0}, \tau=0
$$

The boundary conditions are [26],

$$
\left\{\begin{array}{c}
-\lambda \frac{\partial t}{\partial r}=-h\left(t-t_{f}\right), r=r_{0}, \tau>0(\text { Convection heat exchange }) \\
t(\tau, \infty)=t_{0}, \tau>0(\text { outer boundary })
\end{array}\right.
$$

The interface continuity conditions are,

$$
\left\{\begin{array}{c}
t_{k}\left(\tau, r_{k}\right)=t_{k+1}\left(\tau, r_{k}\right), \tau>0 \\
\lambda_{k} \frac{\partial t_{k}}{\partial r}=\lambda_{k+1} \frac{\partial t_{k+1}}{\partial r}, \tau>0
\end{array}\right.
$$

where $t_{0}, t_{f}, r_{0}$, respectively, represent the initial temperature of rock, humidity of air and radius of tunnel. $h$ represents convective heat transfer coefficient between lining and air.

\subsection{Finite Difference Discretization}

For Formula (1), the spatial diffusion term $\frac{\partial t_{k}}{\partial r} \frac{\partial^{2} t_{k}}{\partial r^{2}}$ and the temperature instability term in time $\frac{\partial t_{k}}{\partial \tau}$. One-dimensional unsteady heat conduction in the time-space region can be discretized according to Figure 1. 


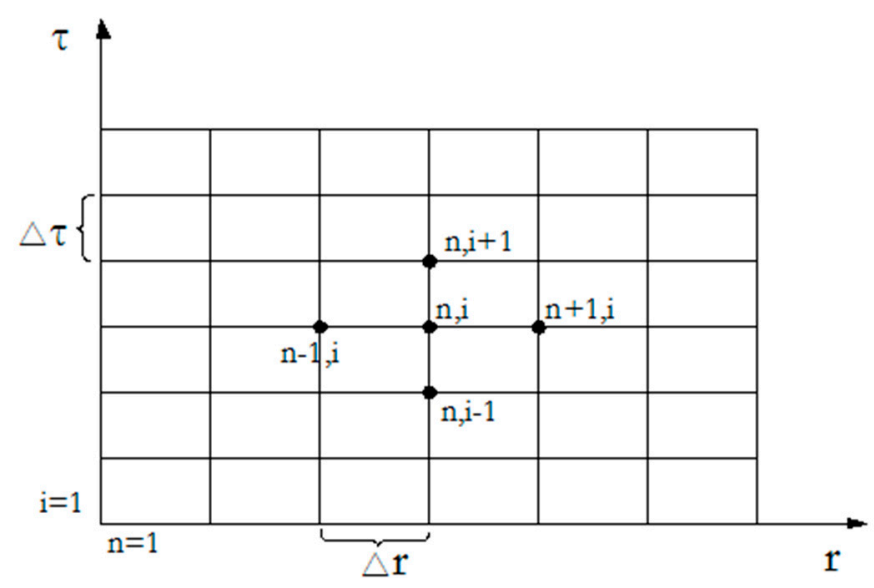

Figure 1. Time-space regional discretization of one-dimensional unsteady heat conduction. Where $r$ is the space coordinate and $\Delta r$ is the space step. $\tau$ represents the time coordinate and $\Delta \tau$ is the time step. $(n, i)$ is the position of a node on the time-space region, and the corresponding temperature is recorded as $t_{n}^{i}$.

\subsection{Algebraic Equations of Physical Quantities of Nodes}

\subsubsection{Discrete Equations for Internal Nodes}

(1) Discretization of diffusion terms

Take the $i$-th time-layer diffusion term node (n, i) as an example, the difference expression is derived. Based on node $(n+1, i)$ and node $(n-1, i)$, the Taylor series expansion of function $(t)$ to node $(n, i)$ is obtained [27].

$$
\begin{gathered}
t_{n+1}^{i}=t_{n}^{i}+\left.\Delta r \frac{\partial t}{\partial r}\right|_{n, i}+\left.\frac{\Delta r^{2}}{2} \frac{\partial^{2} t}{\partial r^{2}}\right|_{n, i}+\left.\frac{\Delta r^{3}}{6} \frac{\partial^{3} t}{\partial r^{3}}\right|_{n, i}+\left.\frac{\Delta r^{4}}{24} \frac{\partial^{4} t}{\partial r^{4}}\right|_{n, i}+\cdots \\
t_{n-1}^{i}=t_{n}^{i}-\left.\Delta r \frac{\partial t}{\partial r}\right|_{n, i}+\left.\frac{\Delta r^{2}}{2} \frac{\partial^{2} t}{\partial r^{2}}\right|_{n, i}-\left.\frac{\Delta r^{3}}{6} \frac{\partial^{3} t}{\partial r^{3}}\right|_{n, i}+\left.\frac{\Delta r^{4}}{24} \frac{\partial^{4} t}{\partial r^{4}}\right|_{n, i}+\cdots
\end{gathered}
$$

The two equations are added and rewritten as an expression as follows:

$$
\left.\frac{\partial^{2} t}{\partial r^{2}}\right|_{n, i}=\frac{t_{n+1}^{i}-2 t_{n}^{i}+t_{n-1}^{i}}{\partial r^{2}}+O\left(\Delta r^{2}\right)
$$

where $O\left(\Delta r^{2}\right)$ represents the $\Delta r$ whose minimum order is 2 in the remainder term. After omitting $O\left(\Delta r^{2}\right)$, the difference expression for the second derivative of the $i$-th time-layer diffusion node is obtained.

$$
\left.\frac{\partial^{2} t}{\partial r^{2}}\right|_{n, i}=\frac{t_{n+1}^{i}-2 t_{n}^{i}+t_{n-1}^{i}}{\partial r^{2}}
$$

Similarly, we obtain the difference expression of the first derivative of the $i$-th time-layer diffusion term.

$$
\left.\frac{\partial t}{\partial r}\right|_{n, i}=\frac{t_{n+1}^{i}-t_{n-1}^{i}}{2 \Delta r}
$$

(2) Discretization of unsteady terms

The Taylor series expansion of function $(t)$ to node $(n, i)$ is obtained.

$$
\left.\frac{\partial t}{\partial \tau}\right|_{n, i}=\frac{t_{n}^{i+1}-t_{n}^{i}}{\Delta \tau}+O(\Delta \tau)
$$


where $O(\Delta \tau)$ represents the $\Delta \tau$ whose minimum order is in the remainder term. After omitting $O(\Delta \tau)$, the difference expression for $\frac{\partial t}{\partial \tau}$ is obtained.

$$
\left.\frac{\partial t}{\partial \tau}\right|_{n, i}=\frac{t_{n}^{i+1}-t_{n}^{i}}{\Delta \tau}
$$

(3) Discrete equations of inner nodes

Substituting Expressions (2), (3) and (4) into Expression (1), the discrete equation of inner nodes is obtained.

$$
t_{n}^{i+1}=\frac{F_{O}}{2}\left[\left(2+\frac{\Delta r}{r_{n}}\right) t_{n+1}^{i}+\left(2-\frac{\Delta r}{r_{n}}\right) t_{n-1}^{i}+\left(\frac{2}{F_{O}}-4\right) t_{n}^{i}\right]
$$

where $F_{O}$ is the grid Fourier number $F_{O}=\frac{a \Delta \tau}{\Delta r^{2}} . r_{n}$ is the distance from the calculating point to the axis of the tunnel. $t_{n}^{i}$ is the temperature of node $n$ at time $i$.

\subsubsection{Establishment of Discrete Equations for Boundary Nodes}

Based on the heat balance method and the Fourier heat conduction law, the energy conservation expression of nodes which represent the unit is obtained. Then, the difference equation is derived from it $[28,29]$.

As shown in Figure 2, Boundary Node 1 represents a unit of width $\Delta \mathrm{r} / 2$ (shaded part in Figure 2). The left side of Node 1 is cooled by the air in the tunnel, where the convection conversion factor is $h$, and the heat is transferred from the unit of Node 2 on the right side. Applying the law of conservation of energy to the unit:

$$
\lambda \frac{t_{2}^{i}-t_{1}^{i}}{\Delta r}\left(r_{0}+\frac{\Delta r}{2}\right) \theta+h\left(t_{f}-t_{1}^{i}\right) r_{0} \theta=\rho c r_{0} \theta \frac{\Delta r}{2} \frac{t_{1}^{i+1}-t_{1}^{i}}{\Delta \tau}
$$

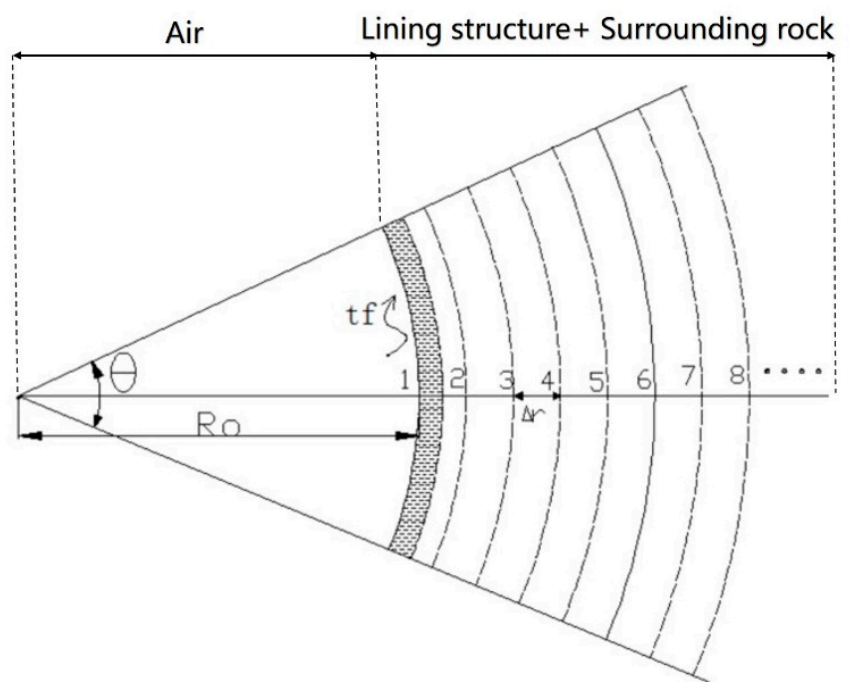

Figure 2. Convective heat transfer boundary node.

The difference equation for convective heat transfer boundary nodes is obtained.

$$
t_{1}^{i+1}=2 F_{O}\left[\left(1+\frac{\Delta r}{2 r_{0}}\right) t_{2}^{i}+B_{i} t_{f}+\left(\frac{1}{2 F_{O}}-1-B_{i}-\frac{\Delta r}{2 r_{0}}\right) t_{1}^{i}\right]
$$

where $B_{i}$ is the number of meshes, $B_{i}=\frac{2 h \Delta \tau}{\rho c \Delta r} \cdot t_{f}$ is the temperature of air in the tunnel. 


\subsubsection{Establishment of Discrete Equations for Composite Interface Nodes}

For the composite interface node, the discrete equation is also derived by the heat balance method. The control unit of the composite interface node contains two kinds of media, and the equivalent thermal properties of the medium need to be calculated.

$$
\begin{gathered}
(\rho c)_{n}=\frac{u(\rho c)_{n-1}+v(\rho c)_{n+1}}{u+v} \\
u=\theta \frac{2 r_{n}-\frac{\Delta r}{2}}{2} \frac{\Delta r}{2}, v=\theta \frac{2 r_{n}+\frac{\Delta r}{2}}{2} \frac{\Delta r}{2}
\end{gathered}
$$

where $(\rho c)_{n}$ is the equivalent $\rho c$ value of the composite interface controlling unit. $(\rho c)_{n-1},(\rho c)_{n+1}$, respectively, represent the $\rho c$ value of two sides of the composite interface. $u, v$ are the volume occupied by the two media in the control element. Based on the energy conservation law:

$$
\lambda_{n-1} \theta \frac{t_{n-1}^{i}-t_{n}^{i}}{\Delta r}\left(r_{n}-\frac{\Delta r}{2}\right)+\lambda_{n+1} \theta \frac{t_{n+1}^{i}-t_{n}^{i}}{\Delta r}\left(r_{n}+\frac{\Delta r}{2}\right)=(\rho c)_{n} \Delta r \theta r_{n} \frac{t_{n+1}^{i+1}-t_{n}^{i}}{\Delta \tau}
$$

The difference equation for composite boundary nodes is obtained.

$$
t_{n+1}^{i}=\frac{\left[\lambda_{n-1}\left(r_{n}-\frac{\Delta r}{2}\right) \frac{t_{n-1}^{i}-t_{n}^{i}}{\Delta r}+\lambda_{n+1}\left(r_{n}+\frac{\Delta r}{2}\right) \frac{t_{n+1}^{i}-t_{n}^{i}}{\Delta r}\right]}{(\rho c)_{n} \cdot \Delta r \cdot r_{n}}+t_{n}^{i}
$$

\section{Field Test}

\subsection{Project Background and Test Objective}

The Sang Zhu Ling tunnel, selected for this study, is a single-track railway tunnel with a total length of approximately $16,449 \mathrm{~m}$. Its maximum overburden is approximately $1480 \mathrm{~m}$. It is located near the Woka garben. Hot spring water and vapor coming from deep in the crust fill the rock cracks and exchange heat with the rock, which causes a rise in the rock temperature, resulting in a highly active geothermal phenomenon. The maximum rock temperature measured in the bore holes is over $89.9^{\circ} \mathrm{C}$, which is the highest temperature of rock on record in a railway tunnel area (Figure 3 ). From the results of the advanced geothermal detection (before construction) in the experimental region (within one kilometer), the initial rock temperature distribution ranged from 46 to $63{ }^{\circ} \mathrm{C}$ along the tunnel line. Based on the field environment conditions, two test locations (NO.1 and NO.2), of which the initial rock temperatures were 47 and $62{ }^{\circ} \mathrm{C}$, were selected for the field test on both surrounding rock temperature and lining temperature (Figure 4 ).
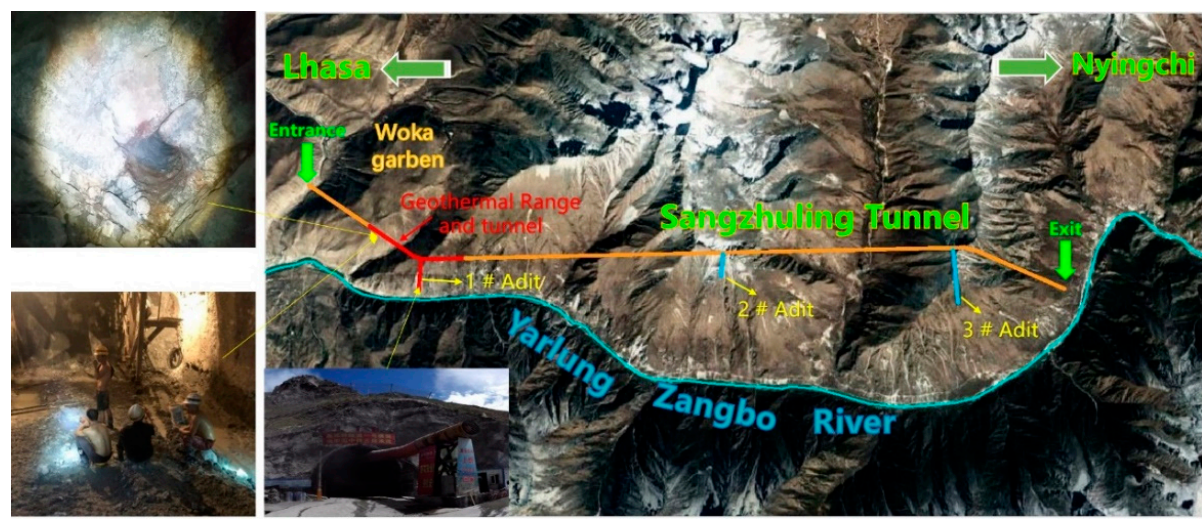

Figure 3. Layout of the Sangzhuling tunnel. 


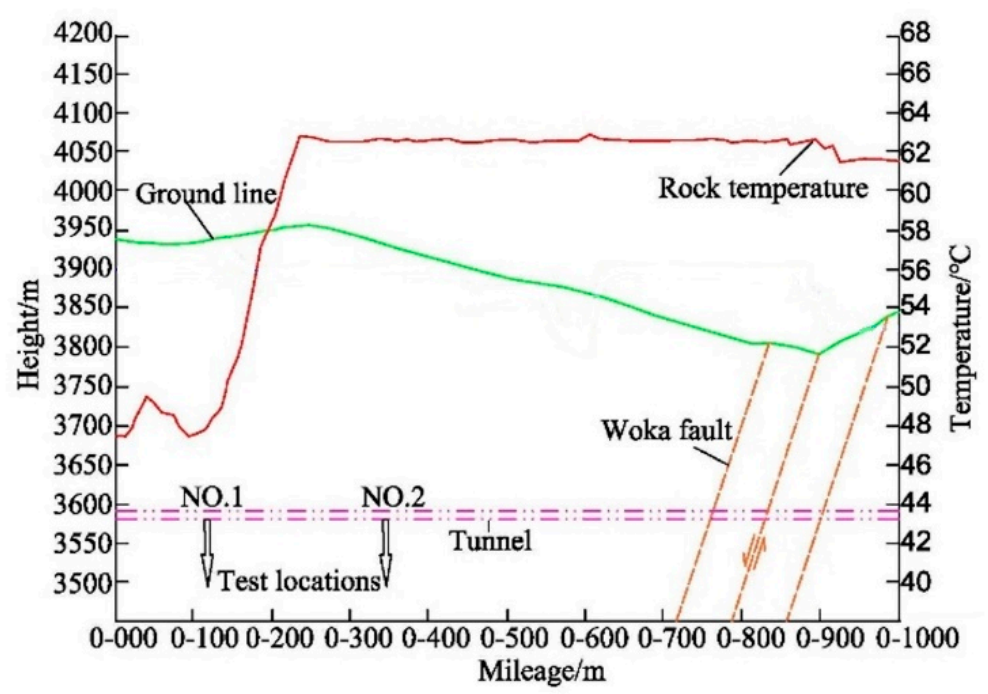

Figure 4. Environmental characteristics of the tunnel.

\subsection{Conditions and Measurements}

\subsubsection{Test of Rock Temperature}

The rock temperature was measured with embedded temperature sensors in the rock hole. As in Figure 5, each hole was excavated by a drilling machine at two sides of the tunnel wall near the test location and each one was $24 \mathrm{~m}$ deep and the hole diameter is $0.22 \mathrm{~m}$. In order to get the variation characteristics of the surrounding rock temperature in the radial direction of the tunnel, the angle between drilling direction and excavation direction is set at $0^{\circ}$. The average of the two test holes' values was taken as the final results of each test location. The temperature sensors were fixed on the test rod and the closer to the tunnel sidewall, the larger the number of temperature sensors were. Finally, the binary slurry was used to grout and seal the heat conducted channel so as to conform to the characteristics of the thermal conduction of the surrounding rock solids. During the construction process, the change in rock temperature with time was recorded by a TST-3826 automatic acquisition instrument during the excavation of the tunnel.

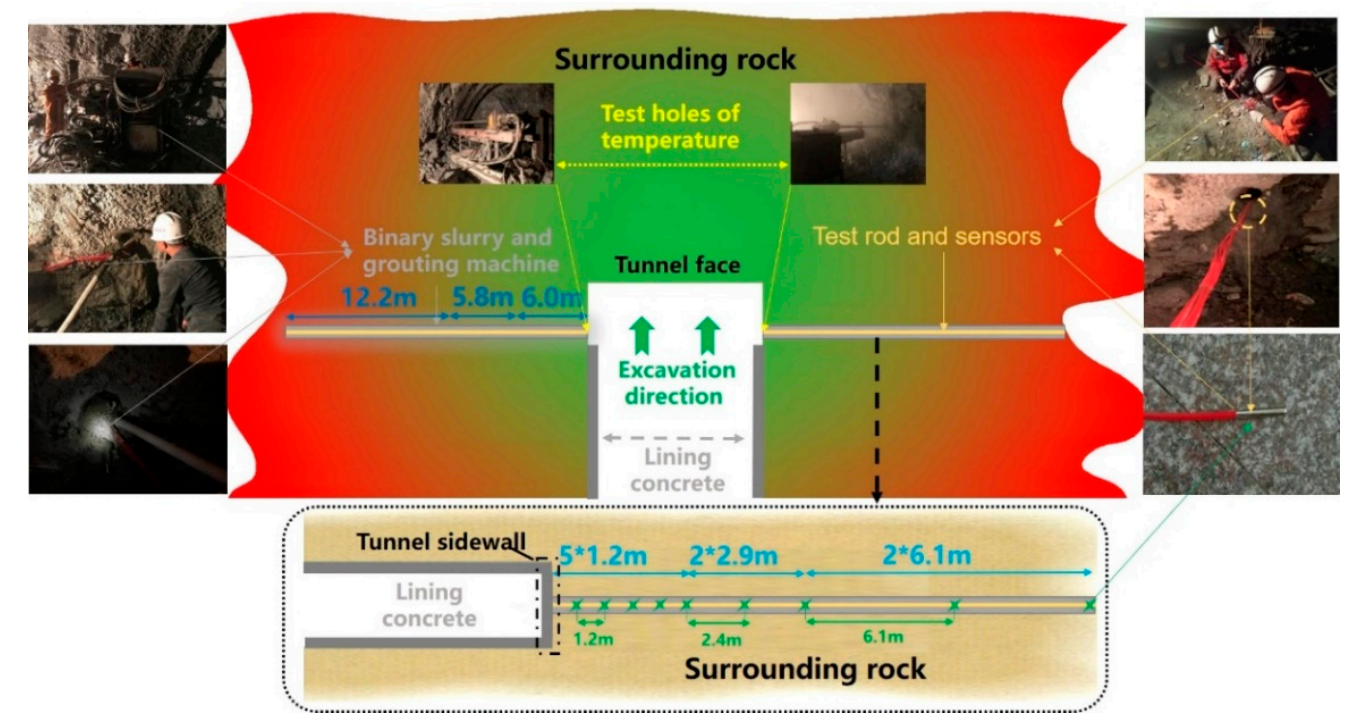

Figure 5. Field test arrangement for surrounding rock temperature. 


\subsubsection{Test of Lining Structure}

The test cross-section was $8.26 \mathrm{~m}$ wide and $10.60 \mathrm{~m}$ high, as shown in Figure 6. Five temperature sensors, which were located at the left wall, the left arch, the vault, the right arch and the right wall, respectively, were attached to each test section in the primary lining. Similarly, two temperature sensors were also installed at the left wall and right wall of the secondary lining. The layout of the measuring point was shown in Figure 7. The acquisition device was a hand-held vibrating strain collector and long-term monitoring was conducted after the installation of the temperature sensors. At the same time, the air temperatures of two test locations were also measured with a hand-held hygrothermograph.

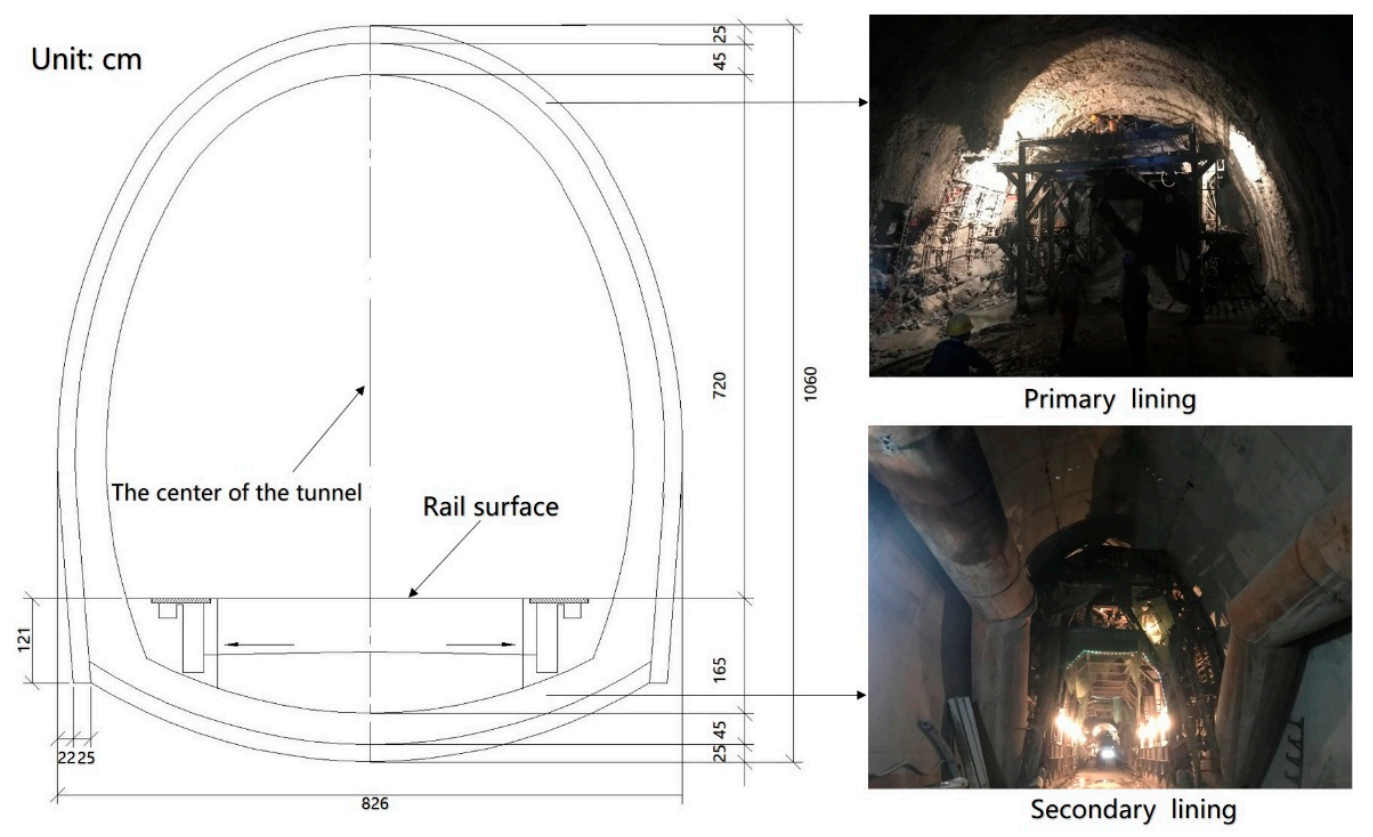

Figure 6. A cross-section of the tunnel.

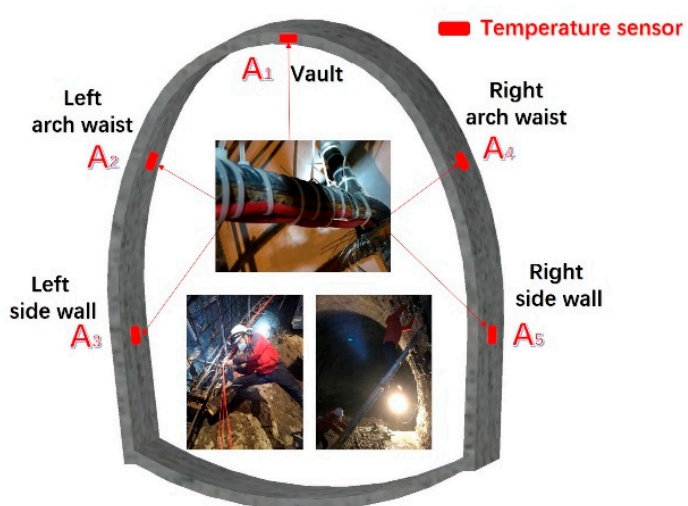

(a)

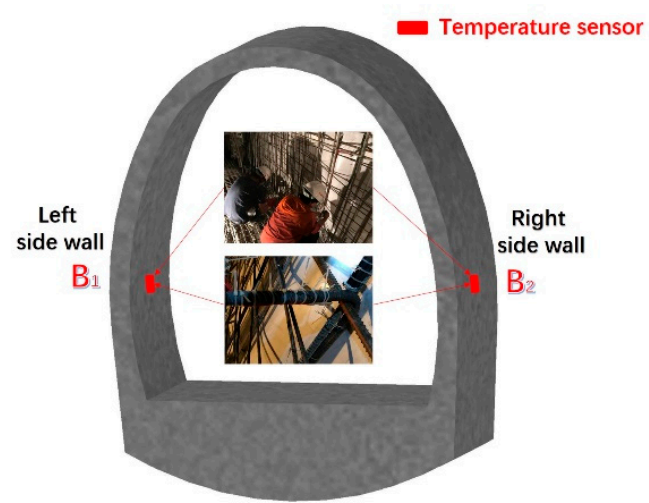

(b)

Figure 7. Layout of temperature monitoring points in lining structure: (a) primary lining and (b) secondary lining.

\subsection{Test Results}

As shown in Figure 8, the temperatures of both surrounding rock and lining structure decrease over the exposure time of excavation, but there exists a relatively large difference in rate and amplitude of decline. Apparently, the reduction of rock temperature near the tunnel sidewall is more significant than that in surrounding rock farther away. In Test Location 1 (the initial rock temperature was $47^{\circ} \mathrm{C}$ ), it can be seen that the temperature of sensors, located 1.2 meters away from the wall, decreased by 
almost $7{ }^{\circ} \mathrm{C}$. The minimum reduction of temperature is $0.3^{\circ} \mathrm{C}$ with sensors at 17.9 meters away from the wall, and the reduction of temperature is below $1{ }^{\circ} \mathrm{C}$ when the distance away from the wall is over six meters. Similar test results can be obtained in Test Location 2 (the initial rock temperature was $62{ }^{\circ} \mathrm{C}$ ). However, the decline of temperature in Test Location 2 ranges from 0.5 to $11^{\circ} \mathrm{C}$ and the reduction of temperature is below $1{ }^{\circ} \mathrm{C}$ when the distance away from the wall is over 8.9 meters. The final steady air temperatures of Test Locations 1 and 2 are $34^{\circ} \mathrm{C}$ and $40{ }^{\circ} \mathrm{C}$, respectively.

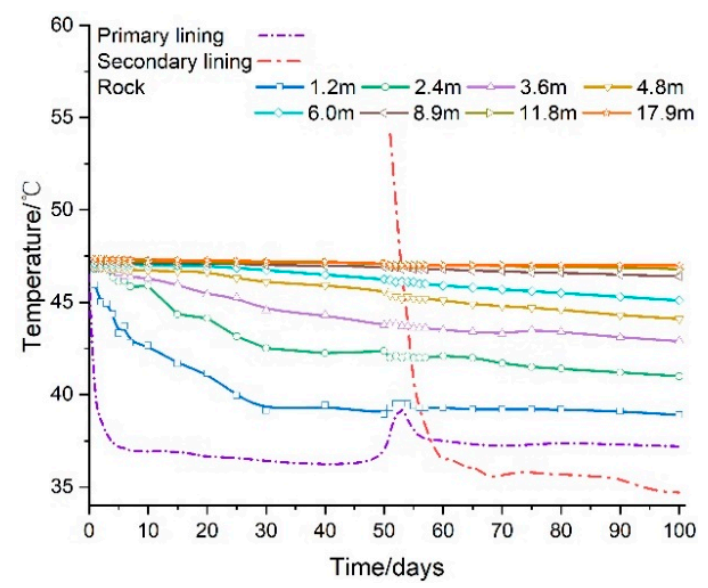

(a)

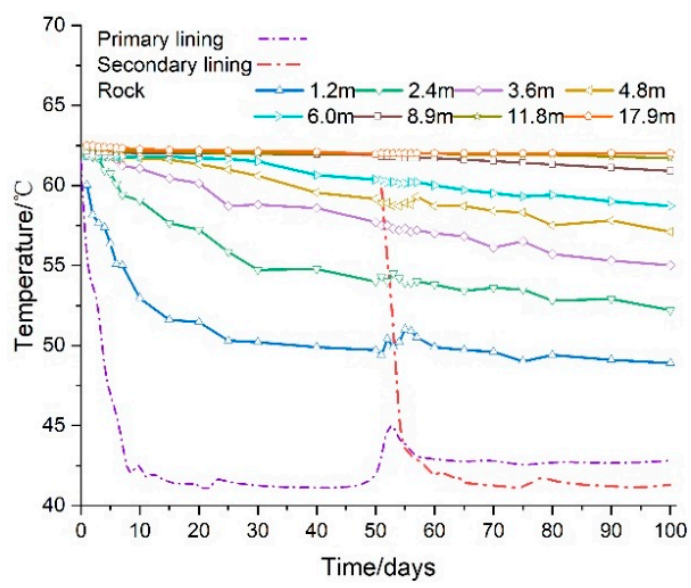

(b)

Figure 8. Variation in temperature of the surrounding rock and lining structure during construction: (a) Test Location 1 and (b) Test Location 2.

As for the temperature of lining structure, it can be seen in Figures 7 and 8 that the temperature distribution of the primary lining was in the range from 37.2 to $46.5^{\circ} \mathrm{C}$ and 42.8 to $61.3^{\circ} \mathrm{C}$, respectively, while the variation ranges of the secondary lining are $35.1-54.1{ }^{\circ} \mathrm{C}$ and $41.3-59.8^{\circ} \mathrm{C}$. Moreover, it appears that there is a temperature rise of $2-3^{\circ} \mathrm{C}$ in the primary lining after the accomplishment of the secondary lining construction. The initial temperature of the concrete decreased from a high level and showed a rapid decline rate within seven days. After almost one week, the decline rate slowed down noticeably at first and then gradually stabilized. It is noticeable, however, that the final temperature of the concrete lining was a little lower than the rock temperature and the temperature of primary lining was a little higher than that of the secondary lining because of heat dissipation at the free face.

MATLAB has a powerful calculating capacity and strict rules of solution, which is significant in equations' solution for its reliability and quickness. According to the formula deduced above and on-site conditions, the temperature field of the high geothermal tunnel is obtained by using MATLAB programming. The equivalent radius was $4.2 \mathrm{~m}$. The time step $\Delta \tau$ was taken as $0.5 \mathrm{~h}$ and the space step is $0.1 \mathrm{~m}$. Calculation thermodynamic parameters of the surrounding rock and lining concrete are shown in Table 1. Iterative calculations are performed by inputting initial parameters, which were obtained from numerous surveys and experiments [30,31].

Table 1. Parameters of heat material.

\begin{tabular}{cccccc}
\hline Materials & $\begin{array}{c}\text { Density } \\
\mathbf{k g} \cdot \mathbf{m}^{\mathbf{- 3}}\end{array}$ & $\begin{array}{c}\text { Thickness } \\
\mathbf{~} \mathbf{m}\end{array}$ & $\begin{array}{c}\text { Thermal Conductivity } \\
\mathbf{W} \cdot\left(\mathbf{m}^{\circ} \mathbf{C}^{-1}\right.\end{array}$ & $\begin{array}{c}\text { Specific Heat } \\
\mathbf{J} \cdot\left(\mathbf{k g}{ }^{\circ} \mathbf{C}\right)^{-\mathbf{1}}\end{array}$ & $\begin{array}{c}\text { Convective Heat Transfer } \\
\text { Coefficient } \mathbf{W} / \mathbf{m}^{\mathbf{2}} \cdot{ }^{\circ} \mathbf{C}\end{array}$ \\
\hline Initial support & 2200 & 0.25 & 2.9 & 960 & 13.96 \\
Second lining & 2200 & 0.45 & 2.9 & 960 & 13.96 \\
Surrounding rock & 2800 & - & 3.2 & 722.1 & - \\
\hline
\end{tabular}




\section{Results and Discussion}

\subsection{Validation of the Calculated Result Against the Field Test Result}

\subsubsection{Surrounding Rock}

Test Locations 1 and 2 were chosen to compare tested results and calculated results of temperature, as shown in Figure 9. It can be revealed that the temperature of the surrounding rock decreased with time. The closer to the side wall, the greater the temperature dropped. The calculated results also reflected the slight rising of rock temperature near the tunnel sidewall after the accomplishment of the secondary lining construction, especially in Test Location 2. The change laws of temperature are consistent with the experimental curve to some extent.

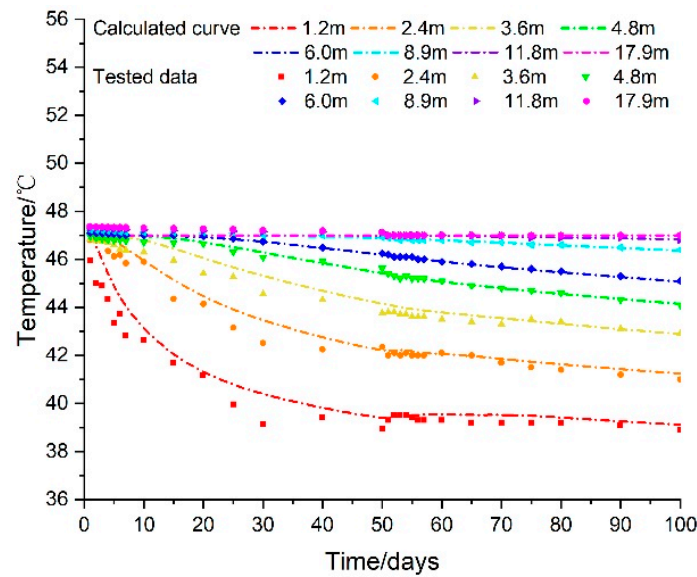

(a)

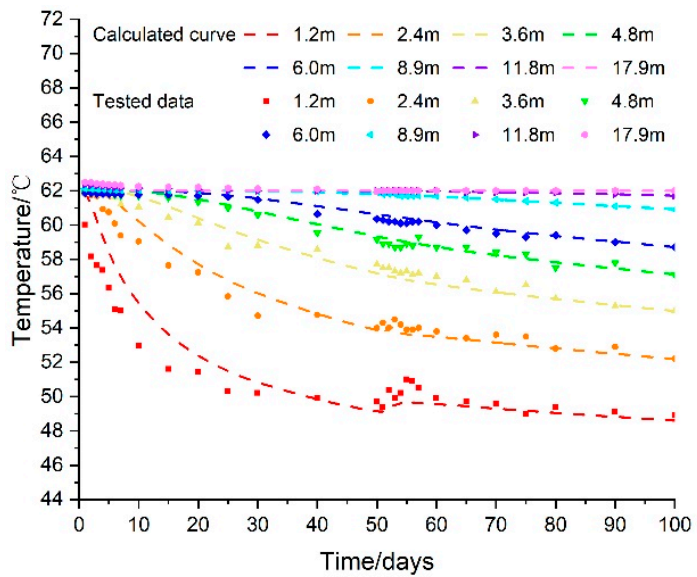

(b)

Figure 9. Comparison of the surrounding rock temperature measured value and predicted value: (a) Test Location 1 and (b) Test Location 2.

However, compared with the test results, the calculated curve deviates from the upside of the experimental curve, but the deviation is small. The difference between tested and calculated results became smaller within the next few weeks. A major reason for this was that in the first few days, tested sections were close to the construction work surface. More cooling measures were taken such as sprinkling water and placing ice cubes to protect the personnel and machinery safety from heat damage. As the working face of the tunnel continued to advance, the heat transfer state of rock and air returned to normal. Nevertheless, the air temperature inside the tunnel was thought of as a constant average value in the prediction model. Moreover, due to the significant effect of cooling measurements on the large temperature difference of the environment, the higher the initial rock temperature, the greater the difference between tested and calculated results.

\subsubsection{Lining Structure}

Figure 10 displays the comparison between calculated and tested results of the primary lining. It can be seen that the calculated results coincide well with the tested results, except that there is some volatility of the tested data. 


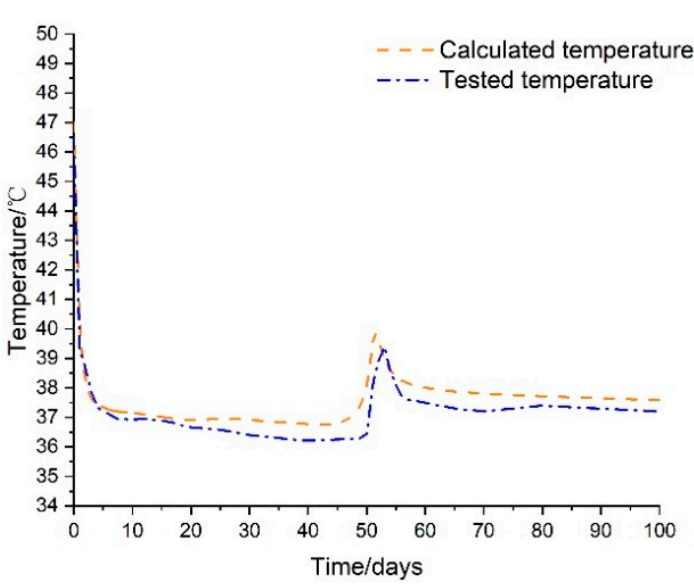

(a)

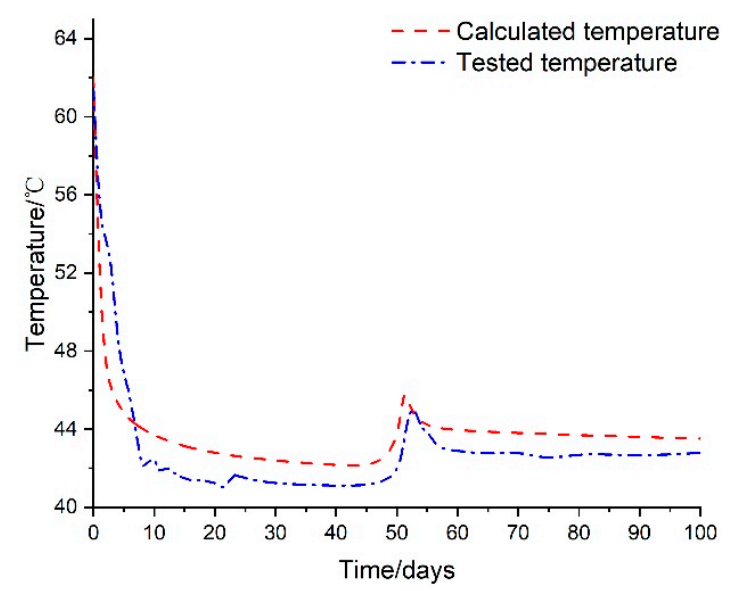

(b)

Figure 10. Comparison of primary lining temperature measured value and predicted value: (a) Test Location 1 and (b) Test Location 2.

The temperature of the primary support decreased dramatically in 5-7 days, and kept close to the air temperature until 50 days. The temperature had declined by more than $90 \%$ of the total amount of temperature drop in seven days. The same phenomenon occurred in the prediction model, where, at the beginning of the 50th day, the temperature of the primary lining increased slightly, and then slowly decreased and stabilized. This was due to the secondary lining on the 50th day, which blocked the convective heat transfer between the primary support and the air in the tunnel, causing the rise of temperature for a short moment. With continuous ventilation, the primary support temperature slowly decreased and approached the air temperature inside the cave again.

Figure 11 presents the temperature of the secondary lining. It revealed that the change trends of calculated and tested results are similar, and the temperature finally reaches a steady. However, the difference between tested and calculated results is large and the difference decreases with time. Apparently, the descending rate of the temperature of the prediction model is faster than that of the tested results, but the results at 90-100 days revealed that the tested temperature is a little lower than that of the calculated results.

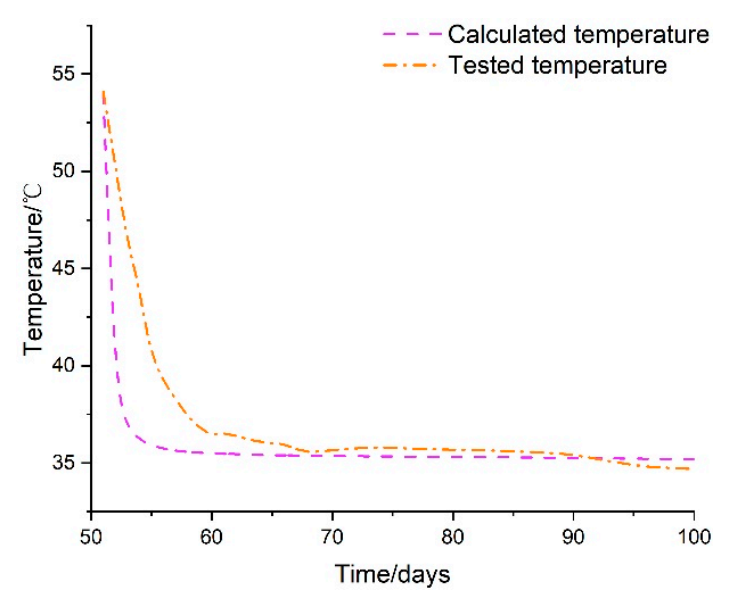

(a)

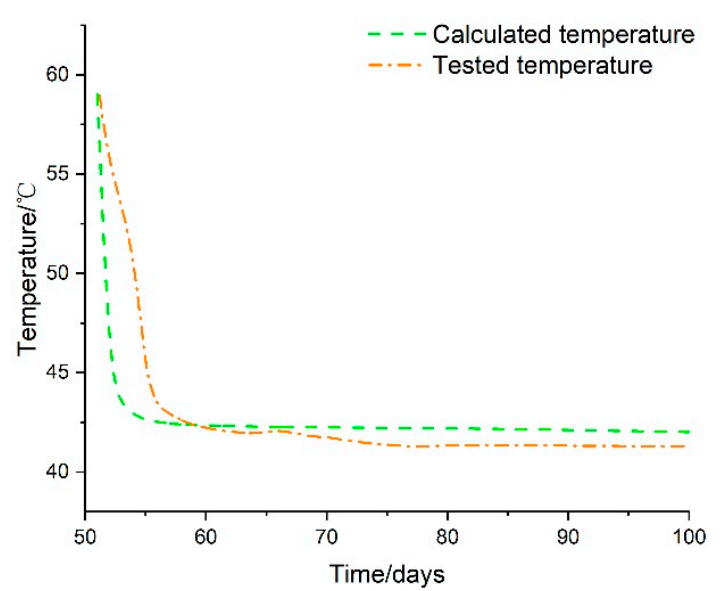

(b)

Figure 11. Comparison of the secondary lining temperature measured value and predicted value: (a) Test Location 1 and (b) Test Location 2.

There is the main reason why the deviation of a lot of heat from the mass concrete structures while solidifying leads to produce temperature change at an early stage. It means that the mass concrete has negative effects on the decline of the secondary lining temperature, which led to deviations between the 
experimental value and the predicted value, especially in the rapid temperature-fall period. Compared with the primary support, the amount of the secondary lining concrete is almost several times than that of the primary support. Therefore, the heat caused by hydration of concrete led to the temperature rising in the lining structure, which is not taken into consideration in the temperature prediction model.

The above research results have proven that the calculated and measured values were in good agreement. However, the existing research results still lack effective cooling design methods [32] to deal with the problem of high-temperature environments in tunnels. Considering the suggested air temperature that was regulated by the Code for Design of Railway tunnel and human health standards [8,33], $28^{\circ} \mathrm{C}$ was chosen to be the value of stable air temperature after ventilation and cooling in the following prediction model.

\subsection{The Partitions of Thermal Response in Surrounding Rock Temperature Field}

The change law of the surrounding rock temperature field was proven to be basically the same by the calculation of heat transfer and temperature change under different initial rock temperature conditions. Moreover, all the temperature change results under different conditions were taken into consideration and summarized. Taking the initial ground temperature at $55^{\circ} \mathrm{C}$ as an example, the variation of the surrounding rock temperature with time at different depths away from the side wall is shown in Figure 12. The decrease of rock temperature near the tunnel sidewall was faster in the first 14 days and then became slower gradually. However, the surrounding rock far away from the tunnel sidewall had no obvious change in temperature. Overall, the rock temperature around the tunnel showed three ways of change and they are corresponding to the three influence areas.

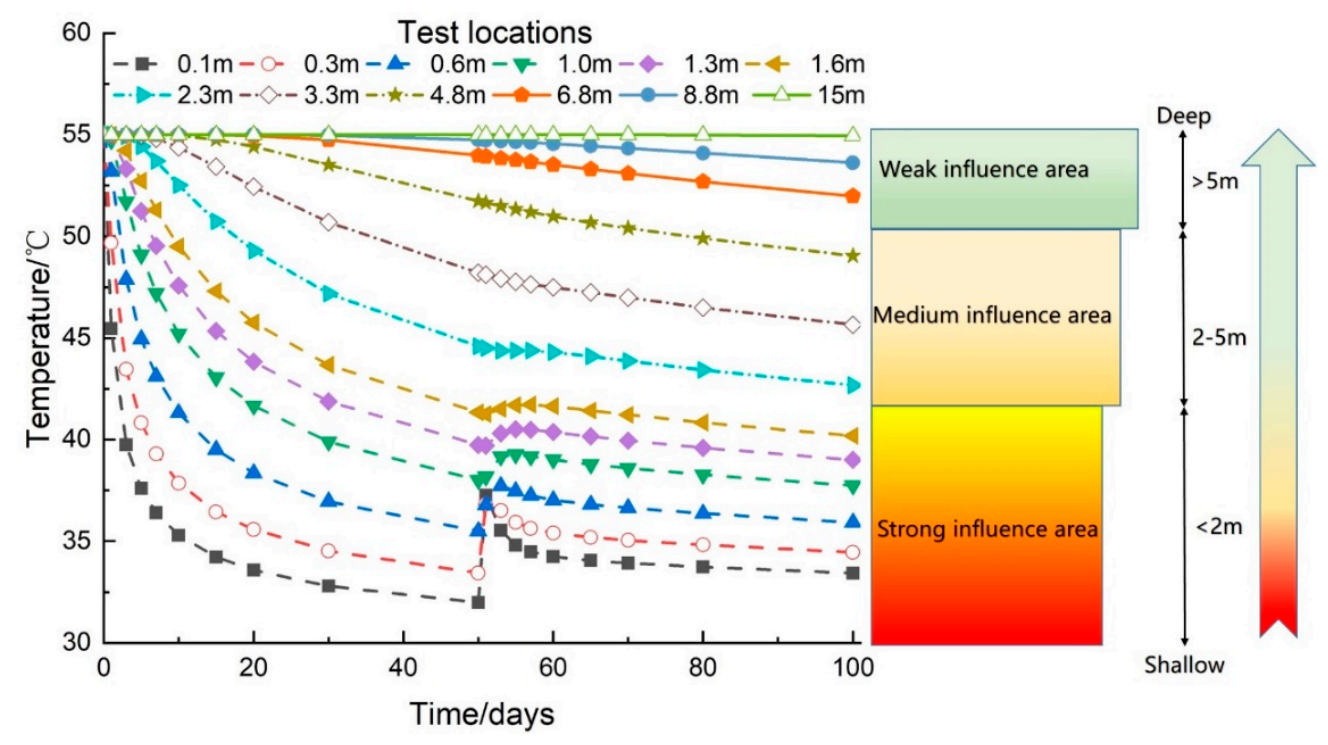

Figure 12. Influence of excavation and cooling measurement on the surrounding rock temperature.

(1) Undulate types (strong influence area): the temperature of the surrounding rock near the tunnel sidewall decreased rapidly in 0-14 days and the cooling rate became slow after 14 days. Affected by the secondary lining at 50 days, the temperature fluctuations slightly rise, and then the temperature slowly decreases. At 50 days, affected by the secondary lining, the temperature increased slightly, and then the temperature slowly decreased.

(2) Decline type (medium influence area): this part is located in the central surrounding rock. The temperature drop curve varies gradually, and the temperature change is little affected by the secondary lining.

(3) Stable type (weak influence area): this part is located in the surrounding rock far away from the tunnel sidewall. The temperature decreased linearly and slowly during the whole process. 
The excavation and the application of the secondary lining had almost no effect on the surrounding rock temperature.

\subsection{Thickness of the Surrounding Rock Heat-Adjusting Layer}

Before excavation of the tunnel, the temperature of the rock mass is in an equilibrium state (initial rock temperature). After tunnel excavation and ventilation, heat transfer occurs from deep to wall at each point in the surrounding rock due to the temperature difference between air and rock mass. The distance from the center of the tunnel to the limit boundary of the heat regulating circle is the disturbance range of the temperature field, that is, the thickness of the heat-adjusting layer. With the extension of ventilation time, the thickness of the heat-adjusting layer keeps increasing, and the increasing rate keeps decreasing, until the disturbance range of the temperature field becomes stable. Through the iterative calculation and comparison of the model, the disturbance range of the temperature field under different initial surrounding rock temperature conditions was obtained. In general, the temperature field of the surrounding rock is stable when the secondary support starts concreting. Moreover, the lining structure may provide some insulation effect because of the difference in thermal conductivity. Therefore, $50 \mathrm{~d}$ was chosen as the final time to calculate the range of thermoregulation effects.

It can be seen in Figure 13 that the maximum radius of the heat regulating circle of the surrounding rock of the tested tunnel is $15.8 \mathrm{~m}$. The thickness of the heat-adjusting layer is the quadric function of initial rock temperature and it increases with the rise of initial rock temperature at an early time, but the curve becomes smooth and slow when the rock temperature exceeds $70^{\circ} \mathrm{C}$. Moreover, the thickness will increase by an average of $2.3 \mathrm{~cm}$ for every degree the temperature increases.

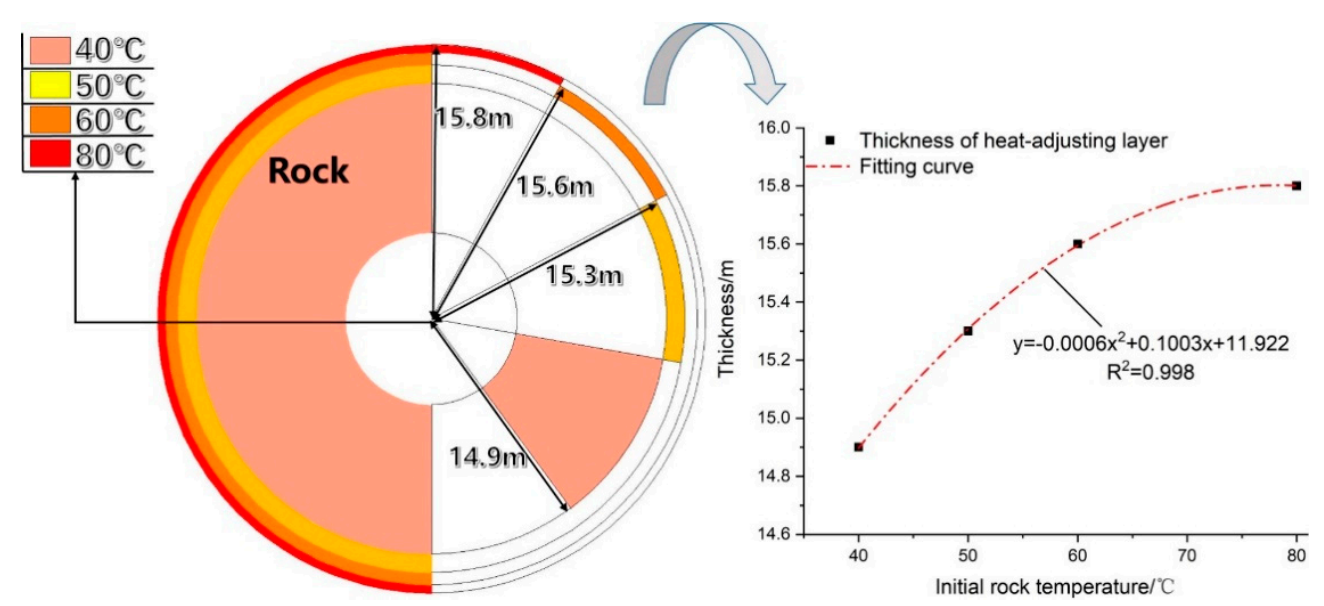

Figure 13. The change rule of heat-adjusting layer thickness under different initial rock temperatures.

As we all know, the heat released by radial surrounding rock mostly depends on the high-temperature rock that is in the range of the heat-adjusting layer. Based on the analysis of the above results and heat transfer theory, the tunnel construction under different initial rock temperature conditions gives off different amounts of heat. As shown in Figure 14, the heat released by the rock is part of the heat source in a high geothermal tunnel. When the temperature is from 40 to $80{ }^{\circ} \mathrm{C}$, the accumulated amount of heat released ranged from 2.29 to $9.93 \mathrm{MJ}$, and it increased linearly with initial rock temperature in 50 days. What was more, the released heat from rock increased, appearing slowly first and fast afterwards, with the thickness of the adjusting layer. The final calculation results may provide basic data for the design of ventilation and a comprehensive cooling system. 

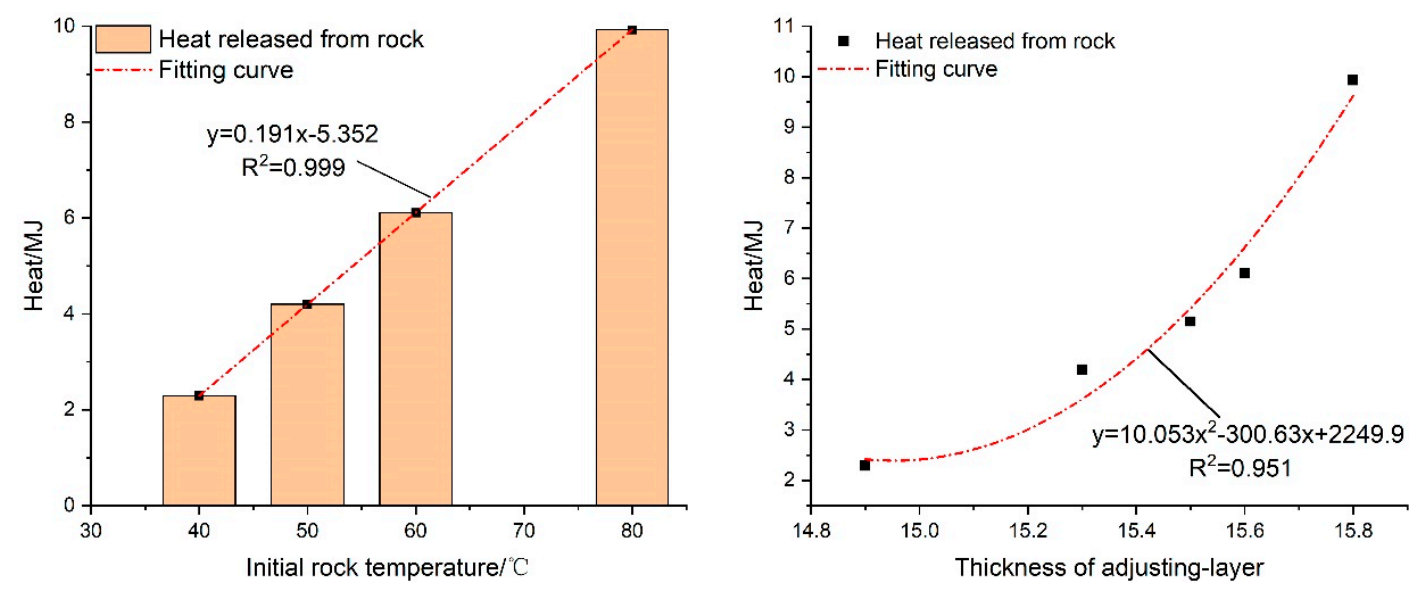

Figure 14. The quantity of released heat from radial surrounding rock. (Left). Rock temperature vs released heat (Right) Thickness of adjusting-layer vs released heat.

\subsection{The Change Rule of Lining Concrete Temperature}

Similarly, the condition of initial rock temperature in the prediction model was set as $40{ }^{\circ} \mathrm{C}, 50^{\circ} \mathrm{C}$, $60{ }^{\circ} \mathrm{C}$ and $80^{\circ} \mathrm{C}$, and the other conditions presented in Section 3 were used. As shown in Figure 8, the initial temperature of the lining concrete decreased from a high level and showed a rapid decline rate within seven days. The initial rock temperature has no significant influence on the time of the rapid temperature decline of the primary lining. When the temperature becomes stable, the higher the initial rock temperature and the higher the lining temperature. The stable temperature of the primary lining ranges from 30 to $36^{\circ} \mathrm{C}$ while the secondary lining is from 29 to $33^{\circ} \mathrm{C}$. The temperature of primary lining has risen by $6.8-7.7^{\circ} \mathrm{C}$ after the completion of the secondary lining in several days and the stable temperature of primary lining is $3.4{ }^{\circ} \mathrm{C}$ higher at most than that of the secondary lining after 100 days.

Therefore, based on the field test results and the calculation samples, the change law of the concrete lining temperature was basically similar to that of the above conclusion when the temperature was below $80^{\circ} \mathrm{C}$. Moreover, as shown in Figure 15, the rising rate of stable temperature is faster with the increase of initial rock temperature. The stable temperature of the four heat transfer mediums in a high geothermal tunnel from high to low were surrounding rock $>$ primary lining $>$ secondary lining > air.

The temperature changing rules of the lining structure revealed that high geothermal may have a significant effect on the concrete structure. In this environment, the concrete is actually under variable high-temperature curing conditions instead of standard curing conditions (at constant $28^{\circ} \mathrm{C}$ ). A previous study revealed that initial higher temperatures generally cause faster development of the shrinkage and the self-induced stresses, which might increase the cracking risks [34,35]. Field conditions caused a $40 \%$ decline in the compressive strength and a 30\% decrease in flexural strength compared with the results measured in the standard curing conditions [36].

Therefore, according to the field tests and calculation results on the concrete temperature, a new model for concrete curing was proposed, which can really reflect the curing process of concrete in high geothermal tunnels. The curing temperature started from the initial high-temperature T1. In the first seven days, the curing temperature was set to decrease at the same rate before it reached $34{ }^{\circ} \mathrm{C}$ on the seventh day. During the period from the 8th to the 28th day, the curing temperature kept constant at $34{ }^{\circ} \mathrm{C}$ (Figure 16). During the entire curing process, the humidity in the test maintained a certain level. 


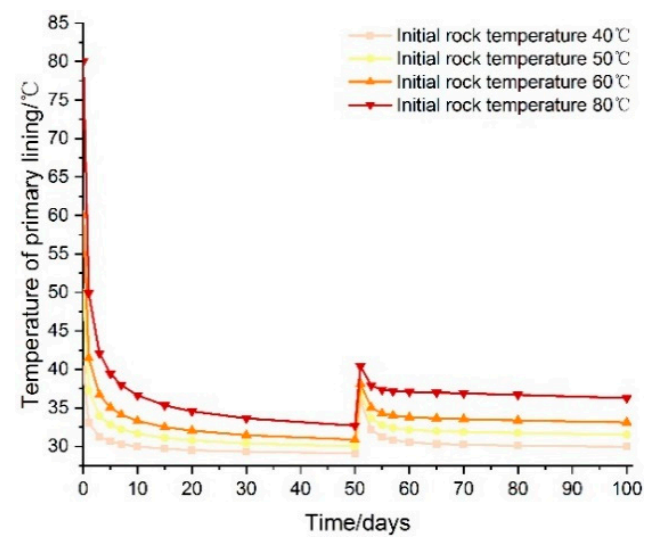

(a)

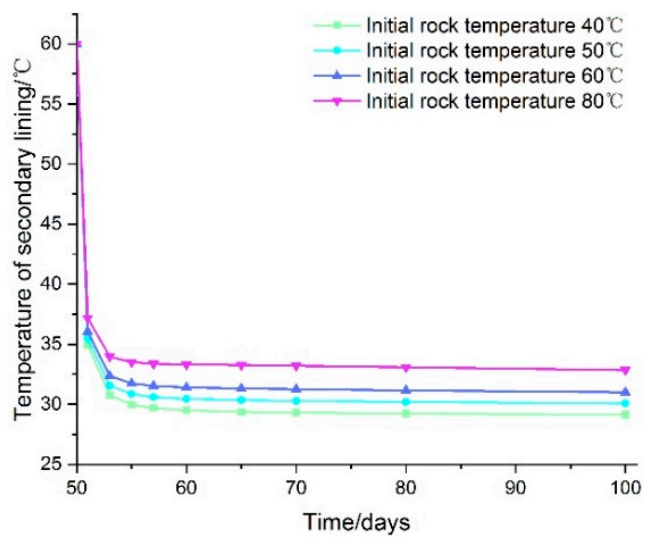

(b)

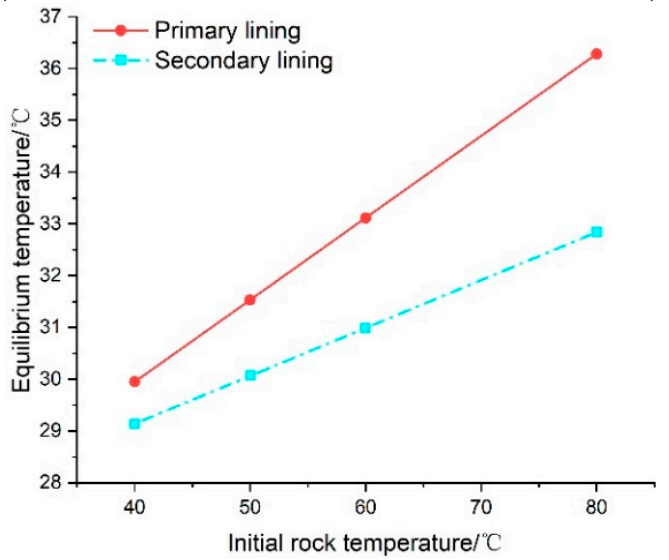

(c)

Figure 15. Temperature variation characteristics of lining structure under different conditions. (a) Temperature change of primary lining. (b) Temperature change of secondary lining. (c). Equilibrium temperature of lining structure under different initial rock temperature.

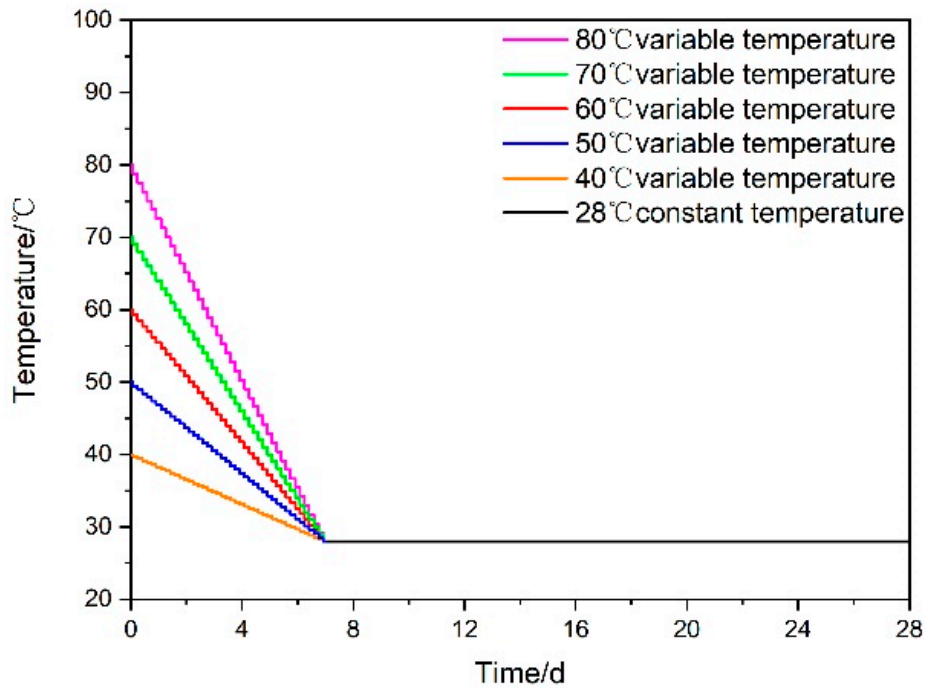

Figure 16. Relationship between curing temperature and curing time of new curing mode.

\subsection{Discussion}

This paper is based on a preliminary study aimed to investigate the distribution of the temperature field and the application of a prediction model in the high geothermal tunnel. It has been proven that the results of the model calculation are consistent with the field experiment data and previous results 
from the numerical simulation $[37,38]$, revealing that the proposed temperature prediction model can better meet the computational requirements for actual engineering tasks. However, many more factors also have a great impact on the distribution of the temperature field, such as lithology and size of the cross-section, especially the cooling measures. The current design of tunnel ventilation mostly emphasizes on the control of the contaminant [39] or smoke spreading of fire [40] and less on cooling ability. Although the traditional cooling design methods at present may not effectively create a proper environment for workers and concrete structures, relative research is already underway [41,42]. This article merely provides a new method of predicting the change of temperature in a high geothermal tunnel and the related regularity conclusion; more detailed research is needed to outline the factors that influence the temperature field in order to improve the present model. Moreover, the conclusion of the temperature impact in this paper may provide help for further design of comprehensive cooling systems and support structures.

\section{Conclusions}

In this paper, a field experiment conducted in a high geothermal tunnel provides several vital conclusions about the distribution and change rules of temperature during the tunnel construction. Based on the finite difference method, the evolution process of a tunnel temperature field was calculated and compared with field test results. The reliability of the finite difference method in analyzing the tunnel heat transfer problem and the tunnel temperature field prediction method was verified. Finally, through the calculation results under different initial surrounding rock temperature conditions, the heat release of rock and actual curing environment of concrete in a high geothermal tunnel are analyzed. The following major conclusions have been obtained:

(1) After the tunnel is excavated and ventilated, the range of low-temperature rock mass extends itself continuously and reaches a new thermal equilibrium state. The closer to the surrounding rock surface, the greater the influence on the rock temperature by ventilating and cooling. Due to the thermal conductivity of rock being bigger than that of the lining structure, the temperature of rock contact with lining rises; farther away from the contact interface, the rock's temperature is approximately constant. According to the distance away from the sidewall, the rock temperature showed three ways of change: undulate type $(<2 \mathrm{~m})$, decline type $(2-5 \mathrm{~m})$ and stable type $(>5 \mathrm{~m})$.

(2) For the rock temperature field before the completion of the secondary lining structure in the high geothermal tunnel, the thickness of the heat-adjusting layer is noted to be second-order polynomial functions of the initial rock temperature. However, the released heat of the surrounding rock increases near linearly.

(3) The temperature of the lining structure dropped sharply within seven days, and then slowly decreased until a stable state. The application of the secondary lining blocked the convective heat transfer between the primary lining and the air in the cave, causing a slight rise of the primary lining temperature, and then the temperature of the primary lining was gradually reduced by the continuous ventilation, finally approaching the air temperature inside the cave again.

(4) During the high-geothermal tunnel construction, the concrete actually cures under the variable high-temperature environment. The real curing temperature decreases with time and becomes stable seven days later.

(5) The calculated data of the in situ investigation of the Sang Zhu Ling tunnel testifies the accuracy of the temperature field calculation method for high geothermal tunnels. The finite difference model proposed in this paper can be used to guide the design of cooling systems and concrete strength for high geothermal railway tunnels.

Author Contributions: Conceptualization, M.W., Y.H., Q.W. and D.L; formal analysis, C.J.; funding acquisition, D.L.; project administration, M.W.; software, Y.W.; writing-original draft, Y.H.; writing-review \& editing, Q.W. All authors have read and agreed to the published version of the manuscript. 
Funding: This research was funded by the National Natural Science Foundation of China (grant numbers: 51578458, 51878568 and 51878567).

Acknowledgments: All authors would like to thank the headquarters of Lhasa-Nyingchi railway construction (grant number: 2017G006-B) for providing support for this field experiment.

Conflicts of Interest: The authors declare no conflicts of interest.

\section{References}

1. Wilhelm, J.; Rybach, L. The geothermal potential of Swiss Alpine tunnels. Geothermics 2003, 32, 557-568. [CrossRef]

2. Mingnian, W.; Yunpeng, H.U.; Jianjun, T.; Qiling, W.; Yicheng, W.; Congyu, D. Experimental study on shear mechanical properties and thermal damage model of shotcrete-rock interfaces under variable high temperatures. Chin. J. Rock Mechan. Eng. 2019, 38, 63-79.

3. Wang, M.; Hu, Y.; Wang, Q.; Tian, H.; Liu, D. A study on strength characteristics of concrete under variable temperature curing conditions in ultra-high geothermal tunnels. Constr. Build. Mater. 2019, 229, 116989. [CrossRef]

4. Ryms, M.; Lewandowski, W.M.; Klugmann-Radziemska, E.; Denda, H.; Wcisło, P. The use of lightweight aggregate saturated with PCM as a temperature stabilizing material for road surfaces. Appl. Therm. Eng. 2015, 81, 313-324. [CrossRef]

5. Geng, J.; Qiang, S.; Zhang, W.; Chao, L. Effect of high temperature on mechanical and acoustic emission properties of calcareous-aggregate concrete. Appl. Therm. Eng. 2016, 106, 1200-1208. [CrossRef]

6. Schackow, A.; Effting, C.; Gomes, I.R.; Patruni, I.Z.; Vicenzi, F.; Kramel, C. Temperature variation in concrete samples due to cement hydration. Appl. Therm. Eng. 2016, 103, 1362-1369. [CrossRef]

7. Wang, Y.; Gao, J.; Xing, X.; Liu, Y.L.; Meng, X.J. Measurement and evaluation of indoor thermal environment in a naturally ventilated industrial building with high temperature heat sources. Build. Environ. 2016, 96, 35-45. [CrossRef]

8. Srinavin, K.; Mohamed, S. Thermal environment and construction workers' productivity: Some evidence from Thailand. Build. Environ. 2003, 38, 339-345. [CrossRef]

9. O'Neal, E.K.; Bishop, P. Effects of work in a hot environment on repeated performances of multiple types of simple mental tasks. Int. J. Ind. Ergon. 2010, 40, 77-81. [CrossRef]

10. Hu, Y.P.; Wang, M.N.; Wang, Q.L.; Liu, D.G.; Tong, J.J. Field test of thermal environment and thermal adaptation of workers in high geothermal tunnel. Build. Environ. 2019, 160, 106174.

11. Zhang, G.; Liu, S.; Xu, Z.; Ye, M.; Chen, R.; Zhang, H.; Yang, J.; Chen, J. The coupling effect of ventilation and groundwater flow on the thermal performance of tunnel lining GHEs. Appl. Therm. Eng. 2017, 112, 595-605. [CrossRef]

12. He, C.; Wu, Z.; Zhu, L. A convection-conduction model for analysis of the freeze-thaw conditions in the surrounding rock wall of a tunnel in permafrost regions. Sci. China Ser. D Earth Sci. 1999, 29, 1-7. [CrossRef]

13. Zhang, X.; Lai, Y.; Yu, W.; Zhang, S. Nonlinear analysis for the three-dimensional temperature fields in cold region tunnels. China Civ. Eng. J. 2003, 35, 207-219.

14. Yuanming, L.; Xuefu, Z.; Wenbing, Y.; Shujuan, Z.; Zhiqiang, L.; Jianzhang, X. Three-Dimensional Nonlinear Analysis for the Coupled Problem of the Heat Transfer of the Surrounding Rock and the Heat Convection between the Air and the Surrounding Rock in Cold-Region Tunnel. Tunn. Undergr. Space Technol. 2005, 20, 323-332. [CrossRef]

15. Zeng, Y.; Liu, K.; Zhou, X.; Lei, F. Tunnel temperature fields analysis under the couple effect of convection-conduction in cold regions. Appl. Therm. Eng. 2017, 120, 378-392. [CrossRef]

16. Zhou, X.; Zeng, Y.; Lei, F. Temperature field analysis of a cold-region railway tunnel considering mechanical and train-induced ventilation effects. Appl. Therm. Eng. 2016, 100, 114-124. [CrossRef]

17. Fan, C.; Zhang, L.; Jiao, S.; Yang, Z.; Li, M.; Liu, X. Smoke spread characteristics inside a tunnel with natural ventilation under a strong environmental wind. Tunn. Undergr. Space Technol. 2018, 82, 99-110. [CrossRef]

18. Li, M.; Wang, C.; Zhang, J.; Yang, S.; Fan, C.; Liu, X. Characteristics of gas phase-controlled flame spread over liquid fuels. Appl. Therm. Eng. 2017, 123, 403-410. [CrossRef]

19. Gao, Z.H.; Ji, J.; Fan, C.G.; Li, L.J.; Sun, J.H. Determination of smoke layer interface height of medium scale tunnel fire scenarios. Tunn. Undergr. Space Technol. Inc. Trenchless Technol. Res. 2016, 56, 118-124. [CrossRef] 
20. Tian, X.; Zhong, M.; Shi, C.; Zhang, P.; Chang, L. Full-scale tunnel fire experimental study of fire-induced smoke temperature profiles with methanol-gasoline blends. Appl. Therm. Eng. 2017, 116, 233-243. [CrossRef]

21. Liang, G.; Lin, J.; Li, S.; Na, S.; Zhang, Y.; Sun, J. Theoretical and experimental study on longitudinal smoke temperature distribution in tunnel fires. Int. J. Therm. Sci. 2016, 102, 319-328.

22. Bo, L.; Ning, Y.Z. A Study on Temperature Distribution of Surrounding Rock and Mechanical Characteristics of Lining of Monglian Tunnel under High Geothermal. Adv. Mater. Res. 2011, 255-260, 2594-2600.

23. Goy, L.; Fabre, D.; Menard, G. Modelling of rock temperatures for deep Alpine tunnel projects. Rock Mech. Rock Eng. 1996, 29, 1-18. [CrossRef]

24. Zhou, X.H. Study on the Influence of Ventilation on Frost Resistance and Reasonable Range of Resistance for Tunnel in Cold Region. Master's Thesis, Southwest Jiaotong University, Chengdu, China, 2012; pp. $29-31$.

25. Zhang, Z.R. Heat Transfer; High Educational Press: Beijing, China, 1989.

26. Yano, H.; Fukutani, S.; Kieda, A. A boundary residual method with heat polynomials for solving unsteady heat conduction problems. J. Frankl. Inst. 1983, 316, 291-298. [CrossRef]

27. Monte, F.D. An analytic approach to the unsteady heat conduction processes in one-dimensional composite media. Int. J. Heat Mass Transf. 2002, 45, 1333-1343. [CrossRef]

28. Wang, Y.; Zhou, G.; Wu, L.; Yang, Z.; Lu, Y. An analytical study of unsteady heat transfer in the rock surrounding a deep airway. Int. J. Min. Sci. Technol. 2012, 22, 411-415. [CrossRef]

29. Richardson, P.D. Unsteady One-Dimensional Heat Conduction with a Nonlinear Boundary Condition. J. Heat Transf. 1964, 86, 298. [CrossRef]

30. Meng, Z.J.; Zhou, Y.M.; Wang, H.Q. Local fractional variational iteration algorithm iii for the diffusion model associated with non-differentiable heat transfer. Therm. Sci. 2016, 20, 781-784. [CrossRef]

31. Liu, J.; Jiang, Y.; Jin, Y.A. Dynamic Model of Heat Transfer through Underground Building Envelope. In Proceedings of the International Conference on Intelligent System Design \& Engineering Application, Changsha, China, 13-14 October 2010.

32. Yi, W.; Zhao, Y.J.; Chan, A.P.C.; Lam, E.W.M. Optimal cooling intervention for construction workers in a hot and humid environment. Build. Environ. 2017, 118, 91-100. [CrossRef]

33. Yi, W.; Chan, A.P.C. Optimizing work-rest schedule for construction rebar workers in hot and humid environment. Build. Environ. 2013, 61, 104-113. [CrossRef]

34. Braun, M.; Zappitelli, M.P.; Villa, E.I.; Rocco, C.G. Application of a linear elastic model to the study of crack stability in concrete dams. Uis. Ing. 2019, 18, 203-211.

35. Mousa, S.; Mohamed, H.M.; Benmokrane, B. Cracking and Crack Control in Circular Concrete Bridge Members Reinforced with Fiber-Reinforced Polymer Bars. J. Bridge Eng. 2019, 24, 23. [CrossRef]

36. Xiao, J.; Fan, Y.; Tawana, M.M. Residual compressive and flexural strength of a recycled aggregate concrete following elevated temperatures. Struct. Concr. 2013, 14, 168-175. [CrossRef]

37. Tong, J.J.; Karakus, M.; Wang, M.N.; Dong, C.Y.; Tang, X.H. Shear strength characteristics of shotcrete-rock interface for a tunnel driven in high rock temperature environment. Geomech. Geophys. Geo-Energy Geo-Resour. 2016, 2, 331-341. [CrossRef]

38. Wang, M.; Tong, J.; Liu, D.; Yu, L.; Zhang, C. Study on support structure system classification of railway tunnel with high rock temperature. China Civ. Eng. J. 2015, 48, 119-125.

39. Chaabat, F.; Creyssels, M.; Mos, A.; Wingrave, J.; Correia, H.; Marro, M.; Salizzoni, P. The effects of solid barriers and blocks on the propagation of smoke within longitudinally ventilated tunnels. Build. Environ. 2019, 160, 11. [CrossRef]

40. Yao, Y.Z.; Li, Y.Z.; Ingason, H.; Cheng, X.D. Numerical study on overall smoke control using naturally ventilated shafts during fires in a road tunnel. Int. J. Therm. Sci. 2019, 140, 491-504. [CrossRef]

41. Han, Q.Y.; Zhang, Y.; Li, K.Q.; Zou, S.H. Computational evaluation of cooling system under deep hot and humid coal mine in China: A thermal comfort study. Tunn. Undergr. Space Technol. 2019, 90, 394-403.

42. Guo, P.Y.; He, M.C.; Zheng, L.G.; Zhang, N. A geothermal recycling system for cooling and heating in deep mines. Appl. Therm. Eng. 2017, 116, 833-839. [CrossRef]

(C) 2020 by the authors. Licensee MDPI, Basel, Switzerland. This article is an open access article distributed under the terms and conditions of the Creative Commons Attribution (CC BY) license (http://creativecommons.org/licenses/by/4.0/). 